\title{
Análisis de la posible influencia de las lesiones sincrónicas en el pronóstico del cáncer colorrectal resecado
}

\author{
A. BORDA MARTÍN, J. M. MARTÍNEZ-PEÑUELA VIRSEDA², M. MUÑOZ NAVAS, \\ C. PRIETO MARTÍNEZ ${ }^{1}$, M. BETÉS IBÁÑEZ, F. BORDA CELAYA ${ }^{1}$
}

Departamento Digestivo. Clínica Universitaria de Navarra. ${ }^{1}$ Servicio de Digestivo. ${ }^{2}$ Servicio de Anatomía Patológica. Hospital de Navarra. Pamplona

\begin{abstract}
ANALYSIS OF POSSIBLE INFLUENCE OF SYNCHRONOUS NEOPLASTIC LESIONS ON PROGNOSIS OF RESECTED COLORECTAL CANCER
\end{abstract}

\section{RESUMEN}

Objetivo: Analizar la relación entre la presencia de lesiones sincrónicas en el cáncer colo-rectal y su pronóstico.

Método: Revisamos 369 cánceres colo-rectales resecados. Comparamos el porcentaje de cirugía aparentemente curativa, la progresión y recidiva tumoral, aparición posterior de cáncer extra-colónico y mortalidad entre los cánceres sincrónicos y el resto. Analizamos los cánceres con adenomas sincrónicos frente al resto de casos. Repetimos el estudio estratificando los casos según su estadio pTNM: estadios 0-I-II versus III-IV.

Resultados: Registramos un 7,6\% de cánceres sincrónicos y un $54,7 \%$ de adenomas sincrónicos. El seguimiento entre los grupos con y sin cáncer sincrónico fue de 70,8 $\pm 22,9$ vs $67,2 \pm 24,5$ meses $(\mathrm{p}=0,55)$. Los cánceres sincrónicos mostraron mayor mortalidad: $35,7 \%$ vs. $14,4 \%$ : $\mathrm{p}=0,006 ; \mathrm{OR}=3,31(1,33-8,13)$, mayor progresión tumoral: 39,3 vs. $19,1 \%: \mathrm{p}=0,011 ; \mathrm{OR}=2,75(1,14-6,56)$ y mayor recidiva: $10,7 \mathrm{vs}$. $3,5 \%: \mathrm{p}=0,096$. Al estratificar según el estadio, los pacientes con estadio 0-I-II y cáncer sincrónico mantuvieron peor pronóstico: mortalidad = 27,7 vs. $8,1 \% \mathrm{p}=0,019 ; \mathrm{OR}=4,45(1,20-15,10)$, progresión tumoral $=$ 27,8 vs. $8,5 \% \mathrm{p}=0,02 ; \mathrm{OR}=4,12(1,14-14,19)$, cáncer extra-colónico $=$ 16,7 vs. $6,4 \% \mathrm{p}=0,095$. No encontramos diferencias entre los casos con y sin adenomas sincrónicos.

Conclusiones: Los cánceres sincrónicos muestran peor pronóstico tras la resección, con mayor tasa de progresión tumoral y mortalidad. Esta diferencia se centra en los casos diagnosticados en estadios 0-I-II, perdiéndose en los estadios III-IV. En nuestra serie, la presencia de adenomas sincrónicos no influye en el pronóstico.

PALABRAS CLAVE: Cáncer colo-rectal. Lesiones neoplásicas sincrónicas. Pronóstico.

\section{ABSTRACT}

Aim: To analyze the relationship between synchronous lesions in patients with colorectal cancer and their prognostic value.

Patients and methods: We have retrospectively reviewed 369 patients with resected colorectal cancer. We compared the rate of apparently curative surgery, progression and tumoral relapse, development of extracolonic cancer and mortality between patients with and without synchronous cancer. Afterwards, we analyzed the same parameters in colorectal cancer with and without synchronous adenomas. Finally, we repeated the analysis after stratification of cancers in 2 groups according to pTNM staging: 0-I-II stage vs III-IV.

Results: We found synchronous adenomas in $54.7 \%$ of our patients and synchronous cancers in $7.6 \%$. Follow-up period of groups with and without synchronous lesions were: $70.8 \pm 22.9$ and $67.2 \pm 24.5$ months $(p$ $=0.55)$ respectivelly. Synchronous cancers showed higher mortality: $35.7 \mathrm{vs} .14 .4 \%: p=0.006 ;$ OR $=3.31$ (1.33-8.13), higher tumoral progression : 39.3 vs. $19.1 \%: p=0.011 ;$ OR $=2.75(1.14-6.56)$ and higher relapse rate: $10.7 \mathrm{vs} .3 .5 \%: p=0.096$. Stratifying according to stage, patients with stage 0-I-II and synchronous cancer showed worse prognosis: mortality $=27.7 v s .8 .1 \%, p=0.019 ;$ OR $=4.45(1.2-15.1)$, tumoral progression $=27.8 v s .8 .5 \%, p=0.02 ; O R=4.12(1.14-14.19)$, and extracolonic cancer $=16.7 \mathrm{vs} .6 .4 \% p=0.095$. There were no statistical differences between cases with and without synchronous adenomas.

Conclusions: Synchronous cancers showed worse prognosis after resection, with higher rate of tumoral progression and mortality. This difference is focused on the cases diagnosed in stage O-I-II, not being found in III-IV. The presence of synchronous adenomas doesn't influence prognosis.

KEY WORDS: Colorectal cancer. Synchronous lesions. Prognosis.

Borda Martín A, Martínez-Peñuela Virseda JM, Muñoz Navas M, Prieto Martínez C, Betés Ibáñez. M, Borda Celaya F. Análisis de la posible influencia de las lesiones sincrónicas en el pronóstico del cáncer colorrectal resecado. An Med Interna (Madrid) 2008; 25: 321 -324.

\section{INTRODUCCIÓN}

El carcinoma colo-rectal sigue manteniendo unas elevadas tasas de mortalidad (1), por lo que constituye en nuestro medio la segunda causa en cuanto a número de fallecimientos por cáncer (2). La Organización Mundial de la Salud ha estimado una tasa de 945.000 casos diagnosticados anualmente en el mundo, de los que 492.000 fallecerán (1). El pronóstico del cáncer colo-rectal se ha relacionado con numerosos factores, probablemente interdependientes (1,3-9).
La multiplicidad de lesiones neoplásicas en forma de adenomas y carcinomas sincrónicos es frecuente en el cáncer de colon y recto (3), por lo que tras su diagnóstico es necesario efectuar un estudio completo del mismo para poder asegurar la resección endoscópico-quirúrgica de todas las lesiones (10). La causa de la multicentricidad tumoral no es bien conocida, postulándose que sea debida a la interacción de múltiples factores, aumentándose así la probabilidad de desarrollar neoplasias en diferentes puntos del colon (3).

La posible influencia de la multicentricidad lesional sobre el

Trabajo aceptado: 10 de marzo de 2008 
pronóstico del cáncer colo-rectal es un tema controvertido. Los estudios publicados muestran unos resultados discordantes, encontrando trabajos que confieren un pronóstico peor (11-13), similar (14-20) o mejor que el de los cánceres sin lesiones neoplásicas sincrónicas (21-23). Estas discrepancias nos han planteado realizar el presente estudio, con el objetivo de revisar una serie de parámetros relacionados con el pronóstico y la supervivencia de los pacientes, analizando sus diferencias entre dos grupos de cánceres colo-rectales con y sin lesiones sincrónicas, para valorar la posible influencia de la multicentricidad lesional con respecto al pronóstico del carcinoma de colon intervenido.

\section{MATERIAL Y MÉTODOS}

Analizamos retrospectivamente una serie de 369 cánceres colo-rectales diagnosticados en dos Centros de nuestra ciudad (Clínica Universitaria y Hospital de Navarra) mediante colonoscopia completa, previa a la cirugía o completada en los 3 meses siguientes a la resección del tumor. Todos los estudios histológicos fueron revisados por un mismo patólogo, con el fin de unificar criterios y evitar diferencias inter-observador. Igualmente se siguieron los mismos criterios en cuanto a indicación y sistemática de tratamiento oncológico pre o postoperatorio. Se excluyeron los cánceres con poliposis adenomatosa familiar o en pacientes con enfermedad inflamatoria intestinal.

Definimos como carcinoma sincrónico a la presencia de dos tumores separados por un segmento de mucosa normal, sin que el segundo sea una propagación o metástasis del primero (24). Contabilizamos como carcinoma sincrónico a los cánceres plenamente desarrollados, así como a los pólipos malignizados, con algún área de carcinoma micro-invasor en su interior. Se ha considerado cáncer sincrónico al que presentaba un estadio tumoral más bajo. Se han incluido como lesión neoplásica sincrónica a los adenomas tubulares, tubulo-vellosos, vellosos y adenomas serrados, excluyéndose los pólipos hiperplásicos.

El estudio se compone de dos partes. En la primera se comparan los factores pronósticos entre los casos de cáncer sincrónico y el resto de la serie. En una segunda parte se analizan los cánceres colo-rectales con lesiones sincrónicas ( carcinomas o adenomas) frente al resto de carcinomas sin este tipo de lesiones. Adicionalmente, se repitieron los análisis estratificando los grupos según el estadio tumoral de la lesión más avanzada en la clasificación pTNM de la American Joint Comittee on Cancer, $5^{\text {a }}$ edición (25). Para facilitar el estudio estadístico se han agrupado los casos en estadios poco avanzados, sin presencia de adenopatías tumorales (estadios 0-I-II) y cánceres más desarrollados (estadios III-IV).

Analizamos los siguientes parámetros:

- Porcentaje de casos en los que se efectuó cirugía aparentemente curativa, definida como los pacientes en los que el cirujano estimaba haber resecado la totalidad del tejido tumoral.

- Progresión tumoral, en forma de adenopatías y/o metástasis, tras el tratamiento inicial.

- Recidiva tumoral local.

- Aparición posterior de otro cáncer extra-colónico

- Tasa de mortalidad al final del seguimiento.

Los resultados de las variables cualitativas se expresan en porcentajes y se comparan mediante los tests de Chi cuadrado y Fisher, determinando la odds ratio (OR) y su índice de confianza al 95\%. Para los datos cuantitativos, se confirmó su distribución normal mediante el test de Kolmogorov-Smirnoff, expresándose como media y desviación típica y analizándose con el test de Student. Se consideraron como significativos los valores de $\mathrm{p}<$ 0,05 .

\section{RESULTADOS}

\section{FRECUENCIA DE LESIONES SINCRÓNICAS}

En 28 pacientes $(7,6 \%$ del global) se registró la presencia de cáncer sincrónico. La totalidad de los cánceres sincrónicos asentaron en pacientes que además tenían adenomas sincrónicos. Ocho pacientes presentaron un segundo cáncer plenamente desarrollado y en 20 se diagnosticó un pólipo malignizado, con áreas de carcinoma microinvasor. Uno de los casos presentó un cáncer más dos pólipos malignizados. En 202 cánceres colo-rectales $(54,7 \%)$ se detectó al menos un adenoma sincrónico. El conjunto de nuestra serie registró un $68,5 \%$ de tumores en estadios poco avanzados (0-I-II), frente al $31,5 \%$ de casos en estadios III-IV.

\section{ANÁLISIS DE PACIENTES CON CÁNCERES SINCRÓNICOS VERSUS RESTO DE CARCINOMAS}

El periodo de seguimiento post-quirúrgico fue similar entre el grupo con cáncer sincrónico: 70,8 $\pm 22,9$ meses y el resto de carcinomas: $67,2 \pm 24,5$ meses, $p=0,55$. Durante el seguimiento el grupo con cáncer sincrónico mostró una mayor tasa de mortalidad: 35,7 versus $14,4 \%: \mathrm{p}=0,006 ; \mathrm{OR}=3,31$ $(1,33-8,13)$, una más elevada progresión tumoral posterior: 39,3 versus $19,1 \%$ : $p=0,011 ;$ OR $=2,75(1,14-6,56)$ y una mayor tendencia a la recidiva: 10,7 versus $3,5 \%, \mathrm{p}=0.096 . \mathrm{El}$ resto de los parámetros estudiados en relación con el pronóstico: índice de cirugía aparentemente curativa y frecuencia de aparición de cáncer extra-colónico, no mostró diferencias significativas entre los grupos con y sin cáncer sincrónicos, como se representa en la tabla I. Al estratificar los carcinomas según su estadio, en los pacientes con estadio 0-I-II y cáncer sincrónico se mantuvo el peor pronóstico, con unas tasas de mortalidad del 27,7 versus $8,1 \%, p=0,019$; OR $=4,45(1,20$ $15,10)$. También fue mayor la progresión tumoral tras la cirugía resectiva: 27,8 versus $8,5 \%, p=0,02$; OR $=4,12(1,14$ $14,19)$, apreciándose una tendencia hacia la mayor frecuencia de aparición de cáncer extra-colónico, $\mathrm{p}=0$ 0,095, sin encontrar diferencias entre el resto de parámetros analizados (Tabla II). No se registró ninguna diferencia significativa entre los grupos con y sin cáncer sincrónico en los pacientes con carcinoma en estadios más avanzados (III-IV) (Tabla III).

TABLA I

ESTUDIO COMPARATIVO GLOBAL ENTRE PACIENTES CON Y SIN CÁNCER SINCRÓNICO COLO-RECTAL

\begin{tabular}{lccc}
\hline Parámetro & Cáncer sincrónico & No cáncer sincrónico & $p$ \\
\hline Mortalidad & $35,7 \%$ & $14,1 \%$ & 0,006 \\
Progresión tumoral & $39,3 \%$ & $19,1 \%$ & 0,01 \\
Recidiva tumoral & $10,7 \%$ & $3,5 \%$ & 0,09 \\
Cirugía aparentemente curativa & $92,9 \%$ & $95,3 \%$ & 0,64 \\
Cáncer extra-colónico & $10,7 \%$ & $5,9 \%$ & 0,40 \\
\hline
\end{tabular}


TABLA II

ESTUDIO COMPARATIVO GLOBAL ENTRE PACIENTES CON Y SIN CÁNCER SINCRÓNICO COLO-RECTAL, CON ESTADIO TUMORAL POCO AVANZADO (ESTADIOS 0-I-II)

\begin{tabular}{lccc}
\hline Parámetro & $\begin{array}{c}\text { Cáncer sincrónico } \\
\text { (Estadios 0-I-II) }\end{array}$ & $\begin{array}{c}\text { No cáncer sincrónico } \\
\text { (Estadios 0-I-II) }\end{array}$ & $P$ \\
\hline Mortalidad & $27,7 \%$ & $8,1 \%$ & 0,019 \\
Progresión tumoral & $27,8 \%$ & $8,5 \%$ & 0,02 \\
Recidiva tumoral & $11,1 \%$ & $3,8 \%$ & 0,18 \\
Cirugía aparentemente curativa & $100 \%$ & $98,3 \%$ & 1 \\
Cáncer extra-colónico & $16,7 \%$ & $5,6 \%$ & 0,095 \\
\hline
\end{tabular}

TABLA III

ESTUDIO COMPARATIVO GLOBAL ENTRE PACIENTES CON Y SIN CÁNCER SINCRÓNICO COLO-RECTAL, CON ESTADIO TUMORAL MÁS AVANZADO (ESTADIOS III-IV)

\begin{tabular}{lccc}
\hline Parámetro & $\begin{array}{c}\text { Cáncer sincrónico } \\
\text { (Estadios III-IV) }\end{array}$ & $\begin{array}{c}\text { No cáncer sincrónico } \\
\text { (Estadios III-IV) }\end{array}$ & $P$ \\
\hline Mortalidad & $50 \%$ & $28,3 \%$ & 0,17 \\
Progresión tumoral & $60 \%$ & $42,5 \%$ & 0,57 \\
Recidiva tumoral & $10 \%$ & $3,8 \%$ & 0,18 \\
Cirugía aparentemente curativa & $80 \%$ & $2,8 \%$ & 0,31 \\
Cáncer extra-colónico & $0 \%$ & $0,9 \%$ & 1 \\
\hline
\end{tabular}

\section{ANÁLISIS ENTRE CÁNCERES COLORRECTALES CON Y SIN ADENOMAS SINCRÓNICOS}

En los 202 casos de cáncer con adenomas sincrónicos, el seguimiento post-quirúrgico fue de 68,3 $\pm 23,7$ meses, similar al de los 167 carcinomas sin pólipos sincrónicos: 66,3 \pm 25,3 meses, $p=0,47$. En el análisis global entre estos dos grupos, no se observaron diferencias significativas entre los casos con adenoma sincrónico (grupo A) y sin adenoma sincrónico (grupo B). Los resultados fueron: Mortalidad: grupo $\mathrm{A}=18,3 \%$, grupo $\mathrm{B}=13,2 \%(\mathrm{p}=0,18)$; Progresión tumoral: grupo $\mathrm{A}=$ $20,3 \%$, grupo $\mathrm{B}=21 \%(\mathrm{p}=0,87)$; Recidiva tumoral: grupo $\mathrm{A}$ $=5,4 \%$, grupo $\mathrm{B}=2,4 \%(\mathrm{p}=0,24)$; Cirugía aparentemente curativa: grupo $\mathrm{A}=96,5 \%$, grupo $\mathrm{B}=93,4 \%(\mathrm{p}=0,17)$ y Cáncer extra-colónico: grupo $\mathrm{A}=10,7 \%$ y grupo $\mathrm{B}=5,9 \%(\mathrm{p}$ $=0,40)$. Tampoco hemos encontrado ninguna diferencia en cuanto al pronóstico al estratificar los carcinomas con y sin adenomas sincrónicos en estadios poco avanzados (0-I-II) (Tabla IV) y más desarrollados (III-IV) (Tabla V).

\section{TABLA IV}

ESTUDIO COMPARATIVO GLOBAL ENTRE PACIENTES CON Y SIN ADENOMA SINCRÓNICO, CON ESTADIO TUMORAL POCO AVANZADO (ESTADIOS 0-I-II)

\begin{tabular}{lccc}
\hline Parámetro & $\begin{array}{c}\text { Adenoma sincrónico } \\
\text { (Estadios 0-I-II) }\end{array}$ & $\begin{array}{c}\text { No adenoma sincrónico } \\
\text { (Estadios 0-I-II) }\end{array}$ & $p$ \\
\hline Mortalidad & $10,1 \%$ & $8,8 \%$ & 0,71 \\
Progresión tumoral & $8,7 \%$ & $11,4 \%$ & 0,47 \\
Recidiva tumoral & $5,1 \%$ & $3,5 \%$ & 0,76 \\
Cirugía aparentemente curativa & $99,3 \%$ & $97,3 \%$ & 0,33 \\
Cáncer extra-colónico & $7,2 \%$ & $5,3 \%$ & 0,52 \\
\hline
\end{tabular}

TABLA V

ESTUDIO COMPARATIVO GLOBAL ENTRE PACIENTES CON Y SIN ADENOMA SINCRÓNICO, CON ESTADIO TUMORAL MÁS AVANZADO (ESTADIOS III-IV)

\begin{tabular}{lccc}
\hline Parámetro & $\begin{array}{c}\text { Adenoma sincrónico } \\
\text { (Estadios III-IV) }\end{array}$ & $\begin{array}{c}\text { No adenoma sincrónico } \\
\text { (Estadios III-IV) }\end{array}$ & $p$ \\
\hline Mortalidad & $36,5 \%$ & $22,6 \%$ & 0,11 \\
Progresión tumoral & $43 \%$ & $41,5 \%$ & 0,62 \\
Recidiva tumoral & $6,3 \%$ & $0 \%$ & 0,12 \\
Ciruǵía aparentemente curativa & $90,3 \%$ & $84,9 \%$ & 0,38 \\
Cáncer extra-colónico & $9,5 \%$ & $1,9 \%$ & 0,12 \\
\hline
\end{tabular}

\section{DISCUSIÓN}

Dadas las elevadas tasas de incidencia y mortalidad que presenta el cáncer colo-rectal, es muy importante el poder determinar los factores que influyen en su pronóstico, especialmente en los pacientes que han sido operados con intención curativa. Los factores con valor pronóstico que se han propuesto son múltiples e incluyen características macro/microscópicas del tumor $(1,5,9)$, alteraciones genéticas $(1,3,7)$, niveles séricos del CEA (6), características y hábitos del paciente como consumo de tabaco e índice de masa corporal $(26,27)$ y factores dependientes del tratamiento aplicado $(1,4)$. De entre todos ellos, el factor pronóstico al que se le atribuye más valor es la estadificación tumoral pTNM, tras el estudio de la pieza de resección (5), ya que el estadio I muestra una supervivencia a los 5 años del $80-95 \%$, frente al 0-7\% del estadio IV (1).

El cáncer colo-rectal presenta con gran frecuencia lesiones multicéntricas, habitualmente pre-malignas: adenomas, o mucho más ocasionalmente carcinomas sincrónicos $(1,3)$. En las publicaciones la frecuencia de los cánceres colo-rectales sincrónicos es muy variable, con un rango entre el 1 y el $14 \%$ $(28,29)$. La serie que analizamos registra una tasa de carcinomas sincrónicos del 7,6\%, que puede considerarse elevada, ya que en la mayoría de los trabajos no se supera el $5 \%$., siendo del $4,8 \%$ en una reciente y amplia casuística multicéntrica nacional (3). Así mismo, nuestra tasa de adenomas sincrónicos $(54 \%)$ está en el límite superior de las referidas en la bibliografía: $29-60 \%(3,4)$.

Es posible que la multicentricidad lesional, expresada en forma de lesiones neoplásicas sincrónicas: adenomas o carcinomas, pueda influir en el pronóstico, y por tanto en la supervivencia del cáncer colo-rectal tras la resección del tumor. En la revisión bibliográfica efectuada, los trabajos referentes al posible efecto pronóstico de las lesiones neoplásicas sincrónicas son discordantes, y en general poco actuales (11-23). Sus discrepancias podrían explicarse en parte por el escaso número de cánceres sincrónicos que presentan la mayoría de las series y por las diferencias en cuanto a los métodos empleados para el diagnóstico y la estadificación (19).

Al igual que otros trabajos que estudian el pronóstico de las neoplasias sincrónicas, analizamos de forma global los tumores, sin hacer diferencias en cuanto a su localización. En los pacientes con cánceres sincrónicos hemos registrado un peor pronóstico, con un evidente incremento de la progresión tumoral tras el tratamiento, una tendencia a la mayor recidiva tumoral y una significativa elevación de la mortalidad durante el seguimiento. Nuestros resultados concuerdan con los de 
otras publicaciones, que constataron un peor pronóstico en el cáncer sincrónico $(11,13,18)$. Por el contrario, otros trabajos encuentran un mejor pronóstico en los tumores sincrónicos $(21,22)$, aunque la mayoría no aprecian diferencias entre cánceres únicos o múltiples $(4,15-20)$. El estudio que, en nuestra opinión, muestra un mayor rigor metodológico es el trabajo efectuado en 1996 en Canadá por Passman (19). Dado que el principal factor pronóstico es el estadio tumoral, compara la supervivencia del cáncer sincrónico efectuando una estratificación de los casos según su estadio. A pesar de que en su serie global, los casos con cánceres sincrónicos correspondieron a tumores más avanzados, al comparar la supervivencia a los 5 años estratificada por estadios, no observa diferencias entre cánceres únicos y sincrónicos (19). En nuestro estudio confirmamos que en los tumores avanzados la mortalidad es similar entre carcinomas sincrónicos y solitarios. Por el contrario, en nuestros pacientes en estadio 0-I-II, el pronóstico y la mortalidad son significativamente peores en el cáncer sincrónico, siendo este subgrupo el que confiere un peor pronóstico a la globalidad del cáncer multicéntrico.

Queremos hacer la consideración de que las tasas de pro- gresión tumoral, recidiva y supervivencia que presentamos pueden no ser representativas de la totalidad de cánceres colorectales. Como en el trabajo Canadiense, la casuística que estudiamos se compone de cánceres colo-rectales confirmados mediante pieza de resección del tumor. Esta condición permite la estadificación pTNM, pero puede conllevar un sesgo con respecto a la globalidad de los tumores, al excluir los casos más evolucionados, inoperables o irresecables. Efectivamente, nuestra serie en su conjunto presenta tan solo un $31,5 \%$ de casos en estadios III o IV, frente al $47-56 \%$ de otros trabajos (3), lo que explica los buenos resultados obtenidos.

Finalmente, hemos estudiado la posible relación entre la presencia de adenomas sincrónicos y el pronóstico de los cánceres. Esta relación ha sido mucho menos estudiada, habiéndose postulado que las lesiones adenomatosas sincrónicas se asociaban con un mejor pronóstico $(13,23)$. En nuestra serie, los factores pronósticos estudiados y la mortalidad han mostrado unas tasas algo más desfavorables en los pacientes con adenomas sincrónicos, pero sin alcanzarse diferencias significativas, tanto en el análisis global como al estratificar los casos en estadios tumorales más y menos avanzados.

\section{Bibliografía}

1. Weitz J, Koch M, Debus J, Höhler T, Galle PR, Büchler MW. Colorectal cancer. Lancet 2005; 365: 153-65.

2. Rodrigo L, Riestra S. Dieta y cáncer de colon. Rev Esp Enferm Dig 2007; 99: 183-9

3. Piñol V, Andreu M, Castells A, Paya A, Bessa X, Jover R. Synchronous colorectal neoplasms in patients with colorectal cancer: predisposing individual and familial factors. Dis Col Rectum 2004; 47: 1192-2000.

4. Finlayson EVA. Effect of Hospital volume on life expentancy after selected cancer operations in older adults: a decision analysis. J Am Coll Surg 2003; 196: 410-7.

5. Jessup JM, Stewart AK, Menck HR. The National Cancer Basa Date report of patterns of care for adenocarcinoma of the rectum 1985-95. Cancer 1998; 83: 2408-18.

6. Slentz K, Senagore A, Hibbert J, Mazier WP, Talbott TM. Can preoperative and postoperative CEA predict survival after colon cancer resection?. Am Surg 1994; 60: 528-31.

7. Garrity MM, Burgart LJ, Mahoney MR, Windschitl HE, Salim M, Wiesenfeld M. et al. Prognostic value of proliferation, apoptosis, defective DNA mismatch repair, and p53 overexpression in patients with resected Dukes'B2 or C colon cancer: a North Central Cancer Treatment Group Study. J Clin Oncol 2004; 22: 1572-82.

8. Gil-Bazo I, Páramo JA, García-Foncillas J. Nuevos factores predictivos y pronósticos en el cáncer colorrectal avanzado. Med Clin (Barc) 2006; 126: 541-8.

9. Guerra A, Borda F, Jimenez FJ, Martinez-Peñuela JM, Larrinaga B. Multivariate analysis of prognostic factors in resected colorectal cancer: a new prognostic index. Eur J Gastroenterol Hepatol 1998; 10: 51-8.

10. Strul H, Kariv R, Leshno M, Halak A, Jakubowicz M, Santo M. The prevalence rate and anatomic location of colorectal adenoma and cancer detected by colonoscopy in average-risk individuals aged 40-80 years. Am J Gastroenterol 2006; 101: 263-65.

11. Bekdash B, Harris, S, Broughton CI, Caffarey SM, Marks CG. Outcome after multiple colorectal tumours. Br J Surg 1997; 84: 1442-4.

12. Copeland EM, Jones RS, Miller LD. Multiple colon neoplasms. Prognostic and therapeutic implications. Arch Surg 1969; 98: 141-3.

13. Chu DZ, Giacco G, Martin RG, Guinee VF. Cancer; 1986: 445-50. The significance of synchronous carcinoma and polyps in the colon and rectum. Cancer 1986; 57: 445-50.

14. Ekelund GR, Pihl B. Multiple carcinomas of the colon and rectum. Cancer 1974; 33: 1630-4.

15. Heald RJ, Bussey HJ. Clinical experiences at St. Mark's Hospital with multiple synchronous cancers of the colon and rectum. Dis Colon Rec- tum 1975; 18: 6-10

16. Kaibara N, Koga S, Jinnai D. Synchronous and metachronous malignancies of the colon and rectum in Japan with special reference to a coexisting early cancer. Cancer 1984; 54: 1870-4.

17. Adloff M, Arnaud JP, Bergamaschi R, Schoegel M. Synchronous carcinoma of the colon and rectum: prognostic and therapeutic implications. Am J Surg 1989; 157: 299-302.

18. Cauvin JM, Boutron MC, Arveux P, Liabeuf A, Grobost O, Faivre J. Multiple colorectal cancers. A population based study. Gastroenterol Clin Biol $1992 ; 16: 16-20$

19. Passman MA, Pmmier RF, Vetto JT. Synchronous colon primaries have the same prognosis as solitary colon cancers. Dis Colon Rectum 1996; 39: $329-34$.

20. Chen HS, Sheen-Chen SM. Synchronous and early metacrhonous colorectal adenocarcinoma: analysis of prognosis and current trends. Dis Colon Rectum 2000; 43: 1093-9.

21. Enker WE, Dragacevic S. Multiple carcinomas if the large bowell: a natural experiment in etiology and pathogenesis. Ann Surg 1978; 187 : $8-11$.

22. Welch JP. Multiple colorectal tumors: an appraisal of natural history and therapeutic options. Am J Surg 1981; 142: 274-80.

23. Mattar M, Frankel P, David D, Clarke KO, Chu DZ, Jiang C. et al. Clinico pathologic significance of syncrhonous and metachronous adenomas in colorectal cancer. Clin Colorectal Cancer 2005; 5: 274-8.

24. Moertel CG, Bargen JA, Dockerty MB. Multiple carcinomas of the large intestine: a review of the literature and a study of 261 cases. Gastroenterology $1958 ; 34$ : 85-98.

25. AJCC (American Joint Committee on Cancer) Cancer Staging Manual, $6^{\text {th }}$ Ed. Greene FL, Page DL, Fleming ID (Eds). Springer-Verlag, New York; 2002. p. 113.

26. Chao A, Thun MJ, Jacobs EJ, Henley SJ, Rodríguez C, Calle EE. Cigarrette smoking and colorectal cancer mortality in the Cancer Prevention Study II. J Natl Cancer Inst 2000; 92: 1888-96.

27. Tamakoshi K, Wakai K, Kojima M, Watanabe Y, Hayakawa N, Toyoshima H. et al. A prospective study of body size and colon cancer mortality in Japan: The JACC Study. Int Obes Relat Metab Disord 2004; 28: 551-8.

28. Fante R, Roncucci L, Di Gregorio C, Tamassia MG, Losi L, Benatti P. et al. Frequency and clinical features of multiple tumors of the large bowell in the general population and in patients with hereditary colorectal carcinoma. Cancer 1996; 77: 2013-21.

29. He JJ. Meta analysis of 2025 cases with multiple primary colorectal carcinoma. Zhongua Wei Chang Wai Ke Za Zhi 2006; 9: 225-9. 